\title{
Uma Abordagem de Classificação de Ovos de Parasitos Intestinais Humanos em Imagens Microscópicas usando Redes Neurais Convolucionais
}

\author{
Bruno Alberto Soares Oliveira \\ $P P G$ em Engenharia Elétrica \\ Universidade Federal de Minas Gerais \\ Belo Horizonte, Brasil \\ brunoalbertobambui@ufmg.br
}

Deborah Aparecida Negrão-Corrêa

Instituto de Ciências Biológicas

Universidade Federal de Minas Gerais

Belo Horizonte, Brasil

denegrao@icb.ufmg.br

\author{
João Marcelo Peixoto Moreira \\ Instituto de Ciências Biológicas \\ Universidade Federal de Minas Gerais \\ Belo Horizonte, Brasil \\ jmpm@ufmg.br
}

\author{
Paulo Ricardo Silva Coelho \\ Instituto de Ciências Biológicas \\ Universidade Federal de Minas Gerais \\ Belo Horizonte, Brasil \\ pauloricardo@ufmg.br
}

\author{
Stefan Michael Geiger \\ Instituto de Ciências Biológicas \\ Universidade Federal de Minas Gerais \\ Belo Horizonte, Brasil \\ smg2012@icb.ufmg.br
}

\author{
Frederico Gadelha Guimarães \\ Machine Intelligence and Data Science \\ Universidade Federal de Minas Gerais \\ Belo Horizonte, Brasil \\ fredericoguimaraes@ufmg.br
}

\begin{abstract}
Resumo-Uma das maiores preocupações da área de saúde pública são causadas pelos parasitos intestinais humanos, que são encontrados em grande parte nos países tropicais. $O$ diagnóstico dessas doenças parasitárias se dá por meio de sintomas fisiológicos e exame fecal. Para realizar esse tipo de exame, faz-se necessário que o profissional seja especialista no assunto, devido a dificuldade do processo, que é considerado lento, propenso a erros e pode causar fadiga ocular no especialista. Portanto, o objetivo desse trabalho é utilizar redes neurais convolucionais para classificar ovos de parasitos intestinais, sendo um sistema de auxílio a tomada de decisão no diagnóstico de um exame de fezes. Foram realizados experimentos empíricos de modo a definir uma arquitetura da rede específica para cada problema. Os resultados obtidos demonstraram uma classificação de $99.9 \%$. A aplicação desenvolvida será parte essencial de um futuro sistema totalmente automatizado.

Palavras-chave-Diagnóstico por Imagens Médicas, Doenças
\end{abstract} Parasitárias, Imagem Microscópica Óptica, Ovos de Parasitos Intestinais, Redes Neurais Convolucionais.

\section{InTRODUÇÃo}

Parasitos são organismos que necessitam de outros organismos, para sobreviver, sendo estes os hospedeiros. Para se manterem vivos, os parasitos utilizam-se de recursos fisiológicos do hospedeiro para se nutrirem e serem capazes de reproduzir. Atualmente estima-se que exista um bilhão de pessoas infectadas por doenças parasitárias em todo o mundo. Sendo que em crianças e pessoas imunodeficientes as infecções podem causar maiores distúrbios físicos ou comportamentais e, nos piores casos, levar indivíduo a morte [1].

Existem três principais filos de parasitos que podem causar doenças em humanos: protozoários, helmintos e ectoparasitos. Em estudos anteriores, foi verificado que alguns helmintos tem maior prevalência em amostras de fezes positivas, são eles: Ancylostoma duodenale, Necator americanus, Ascaris lumbricoides, Enterobius vermicularis, Schistosoma mansoni e Trichuris trichiura, não necessariamente nessa ordem de prevalência. Esses mesmos estudos relataram que a maior prevalência destes parasitos se deve à forma de infecção de cada um deles [2] [3].

O diagnóstico de um paciente com suspeita de infecção parasitológica pode variar de acordo com a disponibilidade do médico e dos recursos que o mesmo possui. O médico se utiliza dos sinais clínicos do paciente e o histórico individual, para avaliar a necessidade de fazer o pedido de um teste diagnóstico para helmintíases. Em grande parte dos casos esse diagnóstico pode ser difícil, por isso, é muito comum que o profissional solicite mais de um tipo de exame.

O exame clínico é o primeiro passo para o diagnóstico, sendo o exame parasitológico de fezes o teste mais comum utilizado para esse tipo de diagnóstico. Especialistas treinados, a partir de seu conhecimento prévio, e com o auxílio de um microscópio, examinam o material fecal do paciente em busca de ovos de parasitos. Salienta-se que todo esse processo é realizado de forma manual pelo especialista, em que é comum acontecer erros de diagnóstico devido ao cansaço, fadiga e falta de experiência do profissional [4] [5].

Visando resolver o problema da falta de especialistas treinados para uma tomada de decisão correta e diminuir o tempo necessário para o diagnóstico a partir do exame parasitológico manual de fezes, é necessário o desenvolvimento de tecnologias que possam ser capazes de automatizar esse processo. A classificação automática de ovos de parasitos em exames fecais permitirá a inspeção de um maior número de amostras com um alto grau de confiabilidade e objetividade. A tecnologia pode 
ser útil, principalmente, nos países onde se tem um alto índice de pessoas infectadas por meio de doenças parasitológicas.

Nos últimos anos o estudo em aprendizado profundo vem se difundindo e se tornando cada vez mais presente no dia a dia da sociedade. Aprendizado profundo pode ser entendido como uma família de métodos de aprendizado de máquina que são baseados em redes neurais artificiais (RNA). Esse tipo de aprendizado pode ser supervisionado, semi-supervisionado ou não supervisionado.

Atualmente, um dos principais algoritmos de aprendizado de máquina são as redes neurais convolucionais, também conhecidas como CNNs. Elas pertencem a uma classe de redes neurais profundas e são constantemente aplicadas em tarefas de classificação e detecção de objetos em imagens [6].

Sendo assim, o objetivo dessa pesquisa foi desenvolver uma solução para reconhecimento automático de ovos de algumas espécies de parasitos intestinais humanos por meio de imagens microscópicas. Para atingir tal objetivo, foi realizado um estudo buscando definir uma topologia de CNN que pudesse fazer parte da solução. Com isso, o sistema desenvolvido poder ser utilizado para auxiliar o especialista no diagnóstico de pacientes com doenças parasitárias.

\section{TRABALhOS RELACIONADOS}

$\mathrm{Na}$ última década vários autores se dedicaram a estudar formas de automatizar esse processo de encontrar e classificar ovos em amostras fecais com o auxílio de um microscópio. Grande parte dos trabalhos até o momento se baseiam em aplicar métodos de processamento digital de imagens e reconhecimento de padrões, buscando encontrar características específicas de cada tipo de ovo de parasito para classificá-lo corretamente.

Em [7] foi estudado um algoritmo utilizando técnicas de processamento digital de imagens e uma RNA. As características morfométricas de ovos de parasitos humanos em amostras fecais foram extraídas de imagens microscópicas através do processamento digital de imagens. Uma RNA classificou as espécies de parasitos com base nessas características. Os autores selecionaram quatro características morfométricas com base em três características morfológicas que representam forma, suavidade da concha e tamanho. Os resultados obtidos mostraram uma taxa de classificação correta média de $86,1 \%$ para a RNA-1 e 90,3\% para a RNA-2.

Uma técnica baseada em momentos invariantes foi proposta em [8]. Foram aplicados alguns métodos de processamento digital de imagens, como redução de ruído, aprimoramento de contraste, segmentação e processo morfológico, que foram utilizados na etapa de extração de características da abordagem usada neste estudo. A técnica apresentada neste trabalho permite classificar 16 diferentes ovos de parasitos a partir de suas imagens microscópicas. As simulações foram realizadas em computador comum no ambiente MATLAB e a taxa geral de sucesso variou em torno de $95 \%$.

Assim como no trabalho [8], em [9] foi desenvolvida uma metodologia baseada em momentos invariantes, combinando-a com a máquina de vetores de suporte multiclasse (MCSVM). O método proposto contém quatro estágios: pré-processamento, extração de recursos, classificação e teste. Foi utilizado o software MATLAB para estimar a taxa de classificação de sucesso da abordagem proposta. Para esse objetivo, o método foi testado usando dados de teste. No final do teste, foi obtido uma taxa geral de sucesso de $97,70 \%$.

No estudo [10] foi proposto um método que pôde segmentar e classificar, a partir de imagens de microscopia com impurezas fecais, as 15 espécies mais comuns de cistos de protozoários, ovos de helmintos e larvas no Brasil. A abordagem desenvolvida explora o formato dos objetos elipsoidais e a transformada imagem-floresta para segmentação de imagens. Os resultados indicam que o método implementado é uma abordagem promissora para a automação completa do diagnóstico de enteroparasitoses.

Em [11] foi mencionado que, por mais que existam alguns métodos de identificação automática de ovos de parasitos, ainda existem algumas espécies que não obtiveram um índice aceitável de acerto, por isso, os autores propõe uma solução que visa automatizar o diagnóstico das 15 espécies mais comuns de enteroparasitos no Brasil, usando uma técnica parasitológica sensível, um microscópio motorizado com câmera digital para aquisição e foco automático de imagens e métodos de análise de imagens. Os resultados indicam que a solução proposta é eficaz e adequada para a rotina de laboratório, na qual o exame passa a ser concluído em alguns minutos.

No artigo [12] foi proposto um software que analisa automaticamente imagens microscópicas e consegue classificar parasitos intestinais. A abordagem é baseada na segmentação e treinamento de um classificador, utilizando a combinação de um sistema neuro-fuzzy e uma RNA. O parasito é primeiro localizado pela transformação circular de Hough e depois extraído para segmentação. Os resultados demonstram uma classificação ótima para cada uma das 20 classes de parasitos, com uma taxa de reconhecimento de $100 \%$.

No estudo [13] foi implementado um sistema médico especialista automatizado e útil para o diagnóstico da parasitose intestinal humana para 20 espécies. O software foi desenvolvido com base em um algoritmo de decisão. Uma base de conhecimento foi construída através de informações obtidas de livros e médicos, com informações referentes à cada doença causada por um parasito. O usuário interage com o sistema, respondendo a perguntas. As informações obtidas são cruzadas com um segundo método que utiliza a transformada circular de Hough e um classificador neuro-fuzzy treinado. O sistema foi avaliado com 60 casos de infecção e comparado ao diagnóstico de dois médicos especialistas. Foi obtido 58 diagnósticos corretos, correspondendo a uma precisão de 96,6\%.

No trabalho [14] foi implementada uma solução para automatizar o diagnóstico de parasitos intestinais através de imagens obtidas de um microscópio conectado a um computador. A abordagem explora a detecção de contorno com base na transformação de wavelets em várias escalas para detectar o parasito. Os contornos ativos são combinados com a transformação Hough para realizar a segmentação e extração 
da imagem do parasito. A ferramenta de classificação é baseada em uma rede neural probabilística. $\mathrm{O}$ algoritmo desenvolvido foi testado em 900 amostras de imagens microscópicas de 15 espécies diferentes de parasitos intestinais. O resultado mostra uma taxa de reconhecimento de $100 \%$ de sucesso.

Neste estudo, é proposto um modelo capaz de classificar ovos de diferentes espécies de parasitos humanos, que são: Ancylostoma duodenale, Necator americanus, Ascaris lumbricoides, Enterobius vermicularis, Schistosoma mansoni e Trichuris trichiura. Distinto dos demais trabalhos na literatura que utilizam transformadas e operações morfológicas com filtros já pré-estabelecidos para extraírem as características de cada espécie de ovo, no modelo desenvolvido foi utilizado redes neurais convolucionais, técnica que ajusta adequadamente na etapa de treinamento, os valores dos pesos dos filtros que realçam as características mais importantes de cada espécie de ovo, para posterior classificação.

\section{REFERENCIAL TEÓRICO}

\section{A. Problema de Interesse}

Atualmente, as técnicas realizadas no setor de parasitologia são exclusivamente manuais, podendo ser influenciadas por variáveis não controláveis como a atenção e a experiência do técnico. A identificação dos ovos de parasitos é determinada microscopicamente, por um profissional habilitado, de acordo com a morfologia dos ovos. A Figura 1 ilustra um ovo da espécie Schistosoma mansoni, marcado por um círculo vermelho, que foi previamente localizado por um especialista.

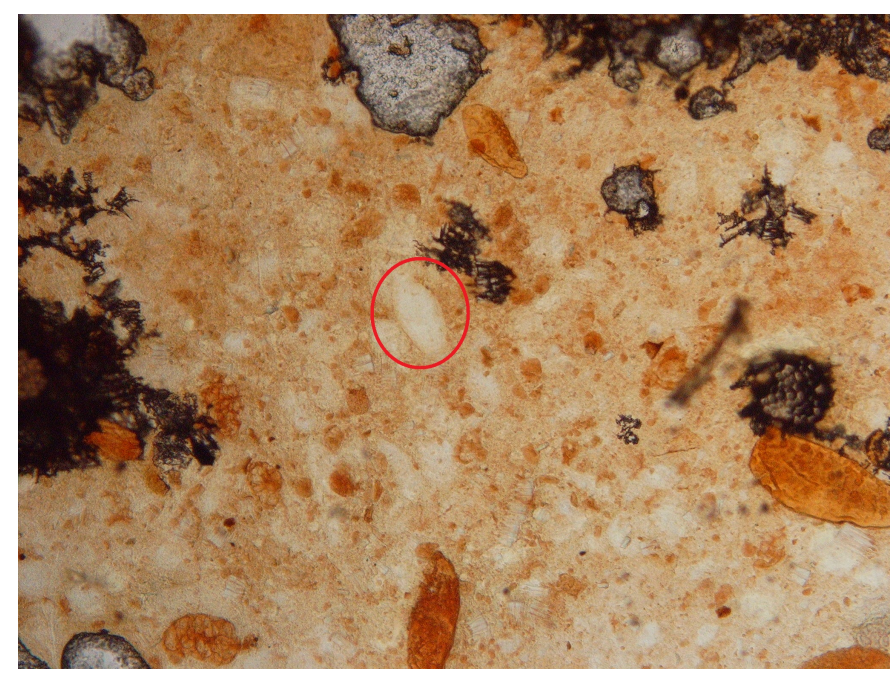

Figura 1. Exemplo de ovo da espécie Schistosoma mansoni

Como pode ser observado na Figura 1, a tarefa de detecção de ovos de parasitos em uma amostra fecal não é trivial para ser feita manualmente. A maior dificuldade que os especialistas enfrentam é o alto acúmulo de sujeira na placa, o que dificulta muito a localização dos ovos. Um outro problema é a quantidade elevada de falsos negativos que existem nos diagnósticos com esse tipo de análise, que é quando o especialista deixa de notar algum ovo na amostra fecal e dá ao paciente um diagnóstico equivocado.

\section{B. Redes Neurais Convolucionais}

A literatura considera que os principais conceitos das redes neurais convolucionais se deram pelo trabalho de [15], que mostrou que os neurônios de alguns animais respondem individualmente a pequenas regiões do campo visual. Foi esse estudo que engajou vários outros trabalhos futuros até chegarmos recentemente na era do aprendizado profundo.

Em 1995, no trabalho de [16], os autores utilizaram esse estudo para arquitetar a primeira rede neural convolucional (CNN). O nome dado é justificado pelo fato de que a rede emprega uma operação matemática chamada convolução, que é um tipo especial de operação linear. CNNs são simplesmente redes neurais que usam a operação de convolução [17].

As CNNs [18], [19] são redes do tipo feedforward e tipicamente possuem as seguintes camadas em sua arquitetura: camadas convolucionais, de subamostragem e densas, que podem ser dispostas de diversas formas a depender do problema abordado.

As camadas de convolução extraem as características mais significativas da imagem. Esse processo é dado através da aplicação de extratores de características na imagem original. Uma vantagem da CNN em relação aos demais métodos é que nessa abordagem é possível obter os valores de extratores de características durante a etapa de treinamento, isto é, não é necessário a participação de um especialista para a escolha das características de treinamento do modelo de classificação.

As camadas de subamostragem, também conhecidas como pooling, segmentam as entradas das camadas anteriores em agrupamentos menores, reduzindo a sensibilidade da saída às pequenas variações e nuances da entrada de dados. Aplicase uma função de pool máximo nos dados de entrada da camada de subamostragem, uma vez que se espera obter as características mais relevantes da imagem.

No fim da arquitetura de uma CNN existe a camada de flattern, que é responsável por redimensionar uma imagem $2 \mathrm{D}$, em um vetor $1 \mathrm{D}$, que posteriormente irão ser os dados de entrada para algum classificador, seja uma perceptron multicamadas (MLP), uma máquina de vetores de suporte (SVM), ou qualquer outro método que possui esse propósito.

O treinamento de uma CNN é similar a outras técnicas mais simples de Redes Neurais Artificiais [20], uma vez que existe uma minimização da função de perda usando Gradiente Descendente. $\mathrm{O}$ erro calculado entre o valor predito e o real é retropropagado pela rede atualizando os parâmetros do modelo, por meio do algoritmo de backpropagation. Desenvolver uma nova arquitetura de CNN para um problema específico não é trivial dada a dependência da configuração de cada camada e dos vários parâmetros do problema a ser resolvido.

Existem algumas arquiteturas de CNN consolidadas e prétreinadas que são aplicadas a problemas de reconhecimento de imagens por meio do aprendizado de transferência, como a MobileNet [21], a Xception [22] e a DenseNet [23]. Neste trabalho optou-se por realizar experimentos empíricos buscando encontrar os melhores parâmetros para definir a arquitetura da rede, uma vez que o problema a ser solucionado é bastante específico. 


\section{Materiais e Métodos}

A metodologia aplicada nessa pesquisa pode ser dividida nas etapas descritas no fluxograma ilustrado na Figura 2 e detalhada na lista de tópicos abaixo.

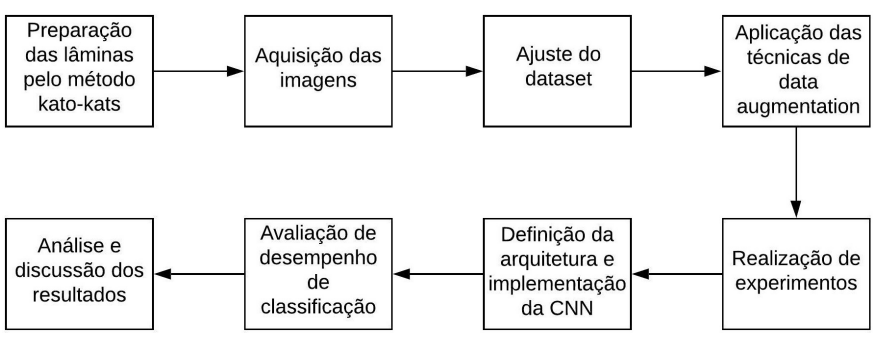

Figura 2. Fluxograma do sistema proposto.

1) Preparação das lâminas pelo método Kato-Katz [24];

2) aquisição das imagens;

3) ajuste do dataset e aplicação das técnicas de aumento de dados;

4) realização de experimentos exaustivos buscando a melhor arquitetura para a rede;

5) definição da arquitetura e implementação da CNN;

6) avaliação de desempenho de classificação;

7) análise e discussão dos resultados.

\section{A. Criação do dataset}

Antes da execução do presente trabalho não havia na literatura um banco de imagens público dos ovos de parasitos das espécies aqui estudadas disponível para avaliação. Tendo disponível o Laboratório de Imunologia de Helmintos da Universidade Federal de Minas Gerais, preparou-se as lâminas pelo método Kato-Katz contendo amostras fecais que posteriormente foram levadas ao microscópio para serem examinadas. Foi utilizado um microscópio clínico da marca Olympus, modelo BX41, ajustado para um zoom de 10 vezes, tendo sido capturadas 66 imagens em RGB com resolução de $2048 \times 1536$ contendo ovos das seguintes espécies de parasitos: Ancylostoma duodenale, Necator americanus, Ascaris lumbricoides, Enterobius vermicularis, Schistosoma mansoni e Trichuris trichiura.

Obtidas as imagens originais e sabendo que o baixo número de imagens pode acarretar um desempenho não satisfatório, pelo fato de que o classificador utilizado ter sido as redes neurais convolucionais, optou-se por empregar técnicas de aumento de dados nessas imagens adquiridas.

Visando criar um conjunto representativo de imagens, com o auxílio de um script desenvolvido na linguagem de programação Python todas as 66 imagens foram cortadas em imagens menores, com dimensões de $200 \times 200$, tendo gerado aproximadamente, após a técnica de corte, 1000 imagens para cada classe. Posteriormente, foram aplicadas rotações de $90^{\circ}$, $180^{\circ}$ e $270^{\circ}$ em cada uma dessas 1000 imagens e que foram separadas manualmente para cada classe. Para finalizar, foi empregado a inversão horizontal na imagem original e em cada imagem rotacionada, obtendo-se ao final, após a utilização de técnicas de aumento de dados um total de 8000 imagens para cada classe das diferentes espécies de parasitos estudadas nesse trabalho.

A maior dificuldade dos especialistas que varrem essas amostras fecais em busca de classificar os ovos não está em saber diferenciar uma classe para outra, mas sim em confundir determinada morfologia de um ovo com alguma sujeira ou impureza contida na amostra fecal. Por esse motivo foram separadas 8000 imagens que não continham ovos de nenhuma espécie, apenas impurezas, visando posteriormente avaliar um classificador binário entre uma determinada espécie e a classe sujeira. Para a classe sujeira, não foram aplicadas técnicas de rotação, essas imagens foram selecionadas após a técnica de corte sob a imagem original de resolução $2048 \times 1536$.

A Figura 3 ilustra exemplos de imagens utilizadas no trabalho desenvolvido. Na primeira coluna, representada pela legenda X.1 estão as imagens originais cortadas. Na coluna representada pela legenda X.2 estão a imagem original com uma rotação de $90^{\circ}$. Para a terceira e quarta coluna, a mesma imagem da primeira coluna tendo sido aplicada rotações de $180^{\circ}$ e $270^{\circ}$, respectivamente. Da quinta à oitava coluna foi aplicado uma inversão horizontal em cada imagem das quatro colunas anteriores.

É possível observar na Figura 3 que as linhas representam cada espécie de ovo de parasito sendo que na primeira linha são ovos da classe Ancilostomídeos, podendo ser das espécies Ancylostoma duodenale ou Necator americanus. Na segunda, terceira, quarta e quinta linha da Figura 3, tem-se ovos das espécies Ascaris lumbricoides, Enterobius vermicularis, Schistosoma mansoni e Trichuris trichiura, respectivamente. A última linha representada pela legenda F.X mostra imagens que não possuem ovos de nenhuma espécie de parasitos, mas que possuem impurezas que podem vir a confundir o especialista durante a análise na amostra de fezes do paciente.

\section{B. Definição da Arquitetura e Implementação da CNN}

Esse estudo tem como objetivo avaliar o desempenho de uma CNN em classificar corretamente ovos de diferentes espécies de parasitos. Para isso, foi utilizado o ambiente de desenvolvimento Google Colab, que é um ambiente de notebooks Jupyter gratuito e disponibilizado pela empresa Google LLC que não requer configuração e é executado na nuvem com suporte para a linguagem de programação Python.

Para a implementação da arquitetura da CNN foi utilizada a biblioteca Keras em sua versão 2.3.0, que possui implementado vários algoritmos de aprendizado de máquina de código aberto escritos em Python e é capaz de funcionar com o TensorFlow, o Microsoft Cognitive Toolkit, o Theano ou o PlaidML. Foi projetada para permitir a implementação rápida de algoritmos de aprendizado profundo, uma vez que tem como premissa facilitar o esforço do desenvolvedor, sendo uma biblioteca modulável e extensível [25].

Foram implementadas duas diferentes arquiteturas de CNN. A primeira com uma saída binária para classificar a presença ou não do ovo de uma determinada espécie na imagem. $\mathrm{O}$ dataset utilizado nessa arquitetura foi composto em duas 


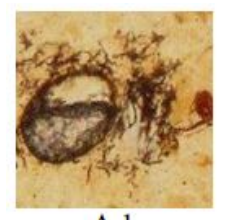

A. 1

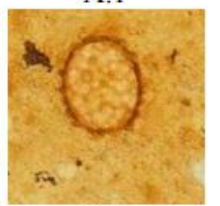

B. 1

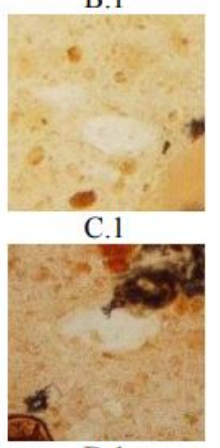

D. 1

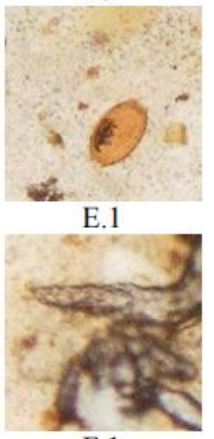

F.1

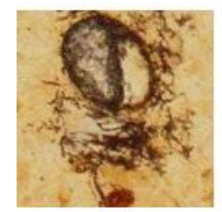

A. 2

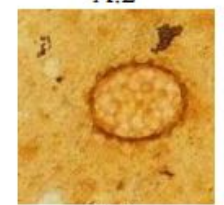

B. 2

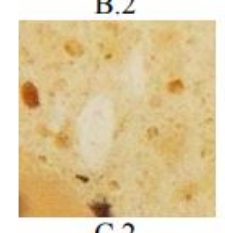

C. 2

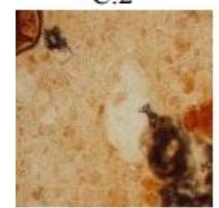

D. 2

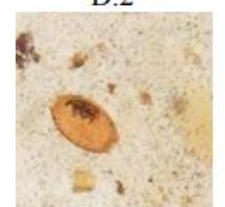

E.2

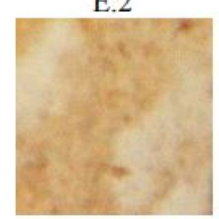

F.2

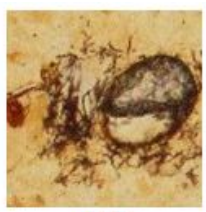

A. 3

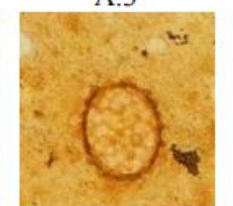

B. 3

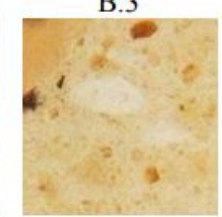

C. 3

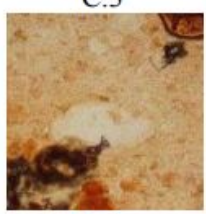

D. 3

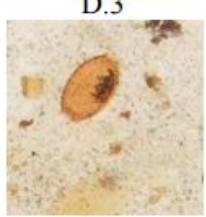

E. 3

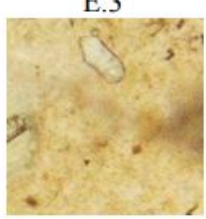

F.3

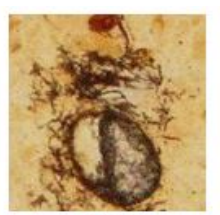

A. 4

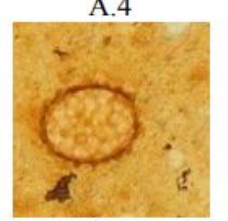

B. 4

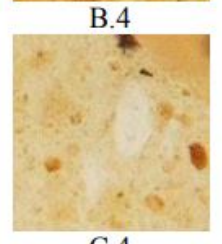

C. 4

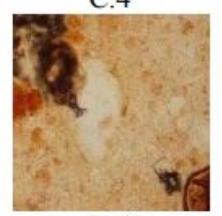

D. 4

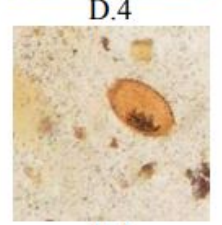

E. 4

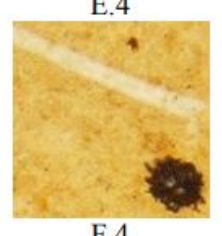

F.4

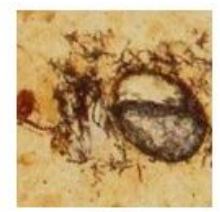

A. 5

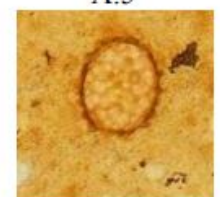

B. 5

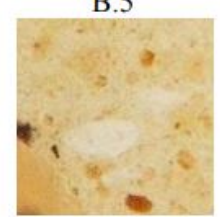

C. 5

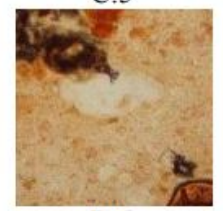

D. 5

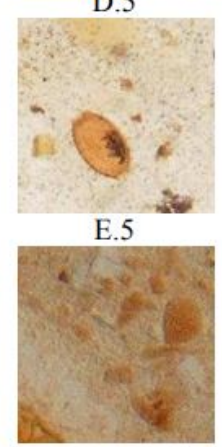

F.5

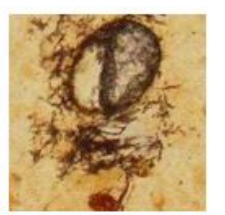

A. 6

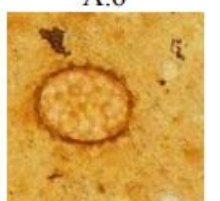

B. 6

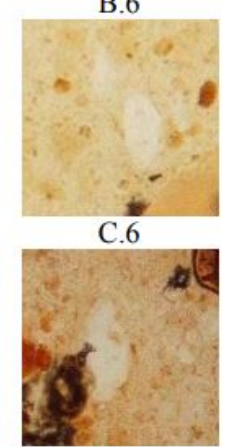

D. 6

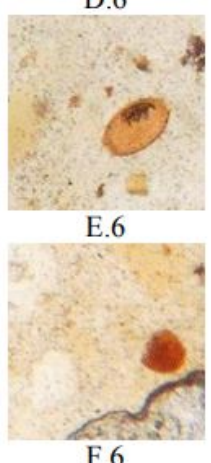

F. 6

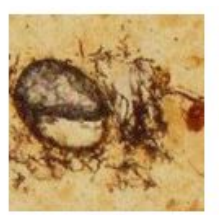

A. 7

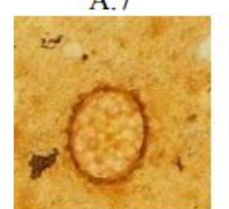

B. 7

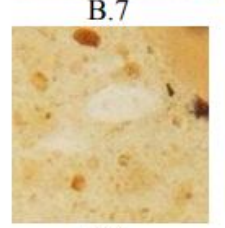

C. 7

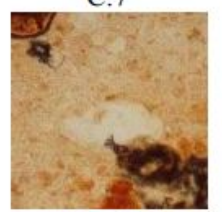

D. 7

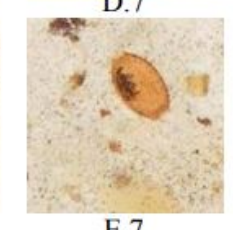

E. 7

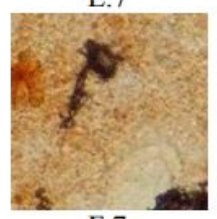

F.7

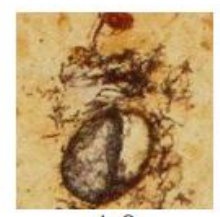

A. 8

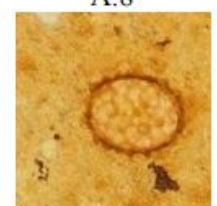

B. 8

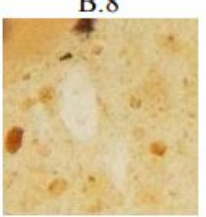

C. 8

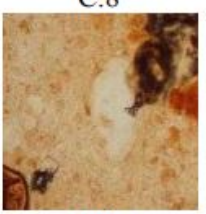

D. 8

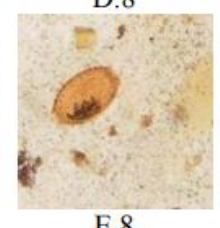

E. 8

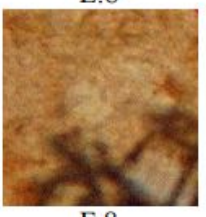

F.8

Figura 3. Exemplos de imagens contidas no dataset utilizado.

classes, sendo uma constituída por imagens que contém ovos de uma única determinada espécie de parasito e uma outra contendo imagens que não possuem nenhum ovo, apenas sujeiras e impurezas. Essa metodologia adotada é justificada pelo fato de que a maior dificuldade do especialista é na identificação dos ovos no meio da sujeira e impurezas em uma amostra fecal.

A segunda arquitetura foi implementada de forma que o método precisou classificar corretamente entre as espécies abordadas nesse estudo, sendo elas: Ancylostoma duodenale, Necator americanus, Ascaris lumbricoides, Enterobius vermicularis, Schistosoma mansoni e Trichuris trichiura.

Testes exaustivos e empíricos foram realizados variando os parâmetros da $\mathrm{CNN}$, como número de épocas, tamanho do lote de imagens, número de camadas de convolução, camadas de pooling, número de extratores de características e seus respectivos tamanhos, utilização ou não de técnicas de regularização, como Batch normalization, Dropout, entre outros. A não utilização de uma arquitetura pré-treinada já consolidada no estado da arte, como a MobileNet [21], a Xception [22] e a DenseNet [23], é justificada pelo fato de que o problema a ser solucionado é bastante específico e requer um estudo mais detalhado.

\section{Avaliação de Desempenho de Classificação}

Para todos os experimentos, o dataset foi dividido aleatoriamente em três conjuntos: treinamento, validação e teste. De um total de $100 \%$ das imagens, o conjunto de treinamento conteve $80 \%$, sendo $20 \%$ para o conjunto de teste. A quantidade de imagens contidas no conjunto de validação foi de $20 \%$ dentre as $80 \%$ sorteadas para o conjunto de treinamento. Para essa divisão foi utilizado o método train_test_split da biblioteca sklearn, que toma o cuidado de manter o balanceamento das classes durante a separação dos conjuntos de treinamento, validação e teste. Tomou-se o cuidado para que, mesmo após realizado o aumento de dados, um mesmo ovo não participasse tanto do treinamento quanto da validação ou do teste. 
Para a análise dos resultados foram realizadas 30 (trinta) simulações para cada técnica proposta utilizando como métricas de avaliação as mais utilizadas para modelos de classificação, sendo elas: acurácia, precisão, revocação e F1score. Foi dado um peso maior ao parâmetro de revocação pois para o problema a ser estudado é importante que o máximo de ovos seja identificado corretamente.

A acurácia indica uma performance geral do modelo, uma vez que dentre todas as classificações, quantas o modelo classificou corretamente. A precisão é calculada através da verificação de quantas classificações estão corretas dentre todas da classe positiva que o modelo obteve. A revocação, também conhecida como sensibilidade ou recall, é obtida pelo seguinte premissa: dentre todas as situações de classe positiva como valor esperado, quantas estão corretas. A F1-Score é a média harmônica entre precisão e revocação. Em consenso com os especialistas do problema, acredita-se que valores superiores a $90 \%$ são considerados interessantes.

\section{Resultados}

\section{A. Arquitetura da $C N N$}

A arquitetura da CNN que trata de um problema de classificação binária é composta por 03 (três) camadas convolucionais, 03 (três) camadas de subamostragem e 03 (três) camadas densas, sendo a ReLU (rectified linear unit) utilizada como função de ativação nas camadas convolucionais e a sigmóide logística na camada de saída. A primeira camada de convolução tem um total de 32 extratores de características, a segunda camada possui 64 extratores e para a terceira camada convolucional foi escolhido um total de 128 extratores, todos com tamanho de $3 \times 3$, uma vez que os ovos representam um objeto específico e pequeno dentro da imagem. A técnica de Batch Normalization foi empregada nas camadas de convolução visando normalizar os valores nos filtros para que o processamento possa ser mais rápido.

Para as camadas densas foi escolhido uma quantidade de 128 neurônios para a primeira camada, seguido por 64 neurônios para a segunda camada e 32 para a terceira. A técnica de Dropout foi utilizada nas camadas densas zerando sempre $20 \%$ da quantidade total dos valores desses neurônios de modo que a rede evite o excesso de ajuste e o modelo não fique super treinado. A Figura 4 ilustra a arquitetura da CNN escolhida para o problema binário.

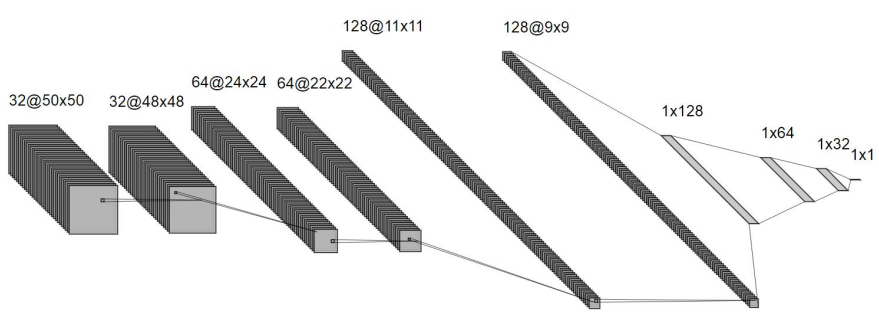

Convolution Max-Pool Convolution Max-Pool

Convolution Max-Pool

Figura 4. Arquitetura da CNN para o problema de classificação binário.
A arquitetura da CNN para classificação multiclasse possui os mesmos parâmetros da arquitetura para o problema de classificação binária, nas camadas de convolução e de subamostragem. As arquiteturas se diferenciam nas camadas densas, uma vez que para o problema de classificação multiclasse foi necessário utilizar um total de 04 (quatro) camadas densas, contendo 128 neurônios em cada uma. A técnica de Dropout utilizada é responsável por zerar $30 \%$ dos valores desses neurônios nas camadas densas. A Figura 5 ilustra a arquitetura da CNN escolhida para o problema multiclasse.

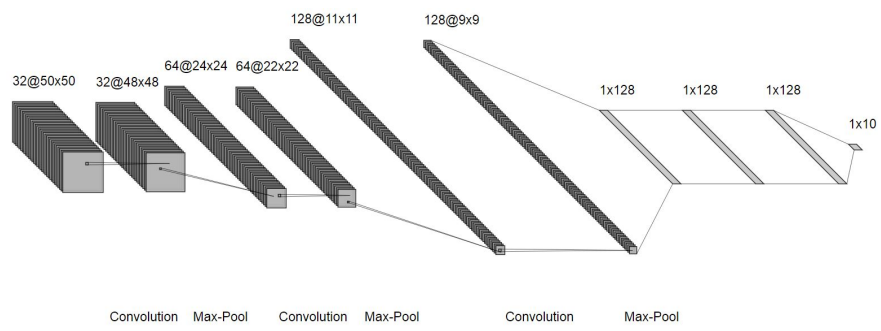

Figura 5. Arquitetura da CNN para o problema de classificação multiclasse.

\section{B. Experimentos}

Para o problema de classificação binária, o dataset utilizado foi composto por oito mil imagens de determinada espécie e oito mil imagens da classe sujeira. Já para o problema de classificação multiclasse foi utilizado um total de $40 \mathrm{mil}$ imagens, oito mil imagens para cada classe, que correspondem a cada espécie de parasito. Ressalta-se que os parasitos Ancylostoma duodenale e Necator americanus estão dentro do grupo dos Ancilostomídeos e seus ovos possuem a mesma morfologia, fazendo-se que ambos pertençam a mesma classe.

Assim como feito por [11], essas simulações foram executadas 30 vezes com conjuntos aleatórios de treinamento, validação e teste, e as Tabelas I, II, III, IV e V mostram a média e o desvio padrão de cada experimento levando em consideração a acurácia, precisão, revocação e F1-score, utilizando a arquitetura definida para o problema binário.

Tabela I

RESULTADOS DOS EXPERIMENTOS REALIZADOS COM OS OVOS DO GRUPO DOS ANCILOSTOMÍDEOS.

\begin{tabular}{|c|c|c|}
\hline & Impurezas & Ancilosmídeos \\
\hline Acurácia & \multicolumn{2}{|c|}{$99.90 \% \quad \sigma \quad 0.003$} \\
\hline Precisão & $100 \% \sigma 0$ & $100 \% \sigma 0$ \\
\hline Revocação & $99.70 \% \quad \sigma 0.005$ & $99.70 \% \sigma 0.005$ \\
\hline F1-score & $99.90 \% \quad \sigma \quad 0.003$ & $99.90 \% \sigma 0.003$ \\
\hline
\end{tabular}

Tabela II

RESULTADOS DOS EXPERIMENTOS REALIZADOS COM OS OVOS DA ESPÉCIE Ascaris lumbricoides.

\begin{tabular}{|c|c|c|}
\hline & Impurezas & Ascaris lumbricoides \\
\hline Acurácia & \multicolumn{2}{|c|}{$100 \% \sigma 0$} \\
\hline Precisão & $100 \% \sigma 0$ & $99.93 \% \sigma 0.002$ \\
\hline Revocação & $99.96 \% \quad \sigma \quad 0.001$ & $100 \% \sigma 0$ \\
\hline F1-score & $100 \% \sigma 0$ & $100 \% \sigma 0$ \\
\hline
\end{tabular}


Tabela III

RESULTADOS DOS EXPERIMENTOS REALIZADOS COM OS OVOS DA ESPÉCIE Enterobius vermicularis.

\begin{tabular}{|c|c|c|}
\hline & Impurezas & Enterobius vermicularis \\
\hline Acurácia & \multicolumn{2}{|c|}{$99.26 \% \quad \sigma 0.008$} \\
\hline Precisão & $99.46 \% \quad \sigma \quad 0.014$ & $99.13 \% \quad \sigma \quad 0.007$ \\
\hline Revocação & $99.13 \% \quad \sigma 0.008$ & $99.50 \% \quad \sigma 0.014$ \\
\hline F1-score & $99.26 \% \sigma 0.008$ & $99.26 \% \sigma 0.009$ \\
\hline
\end{tabular}

Tabela IV

RESULTADOS DOS EXPERIMENTOS REALIZADOS COM OS OVOS DA ESPÉCIE Schistosoma mansoni.

\begin{tabular}{|c|c|c|}
\hline & Impurezas & Schistosoma mansoni \\
\hline Acurácia & \multicolumn{2}{|c|}{$98.63 \% \sigma 0.016$} \\
\hline Precisão & $98.63 \% \sigma 0.025$ & $98.63 \% \quad \sigma 0.019$ \\
\hline Revocação & $98.63 \% \quad \sigma \quad 0.021$ & $98.63 \% \quad \sigma \quad 0.027$ \\
\hline F1-score & $98.60 \% \sigma 0.017$ & $98.63 \% \sigma 0.016$ \\
\hline
\end{tabular}

Tabela V

RESULTADOS DOS EXPERIMENTOS REALIZADOS COM OS OVOS DA ESPÉCIE Trichuris trichiura.

\begin{tabular}{|c|c|c|}
\hline & Impurezas & Trichuris trichiura \\
\hline Acurácia & \multicolumn{2}{|c|}{$100 \% \sigma 0$} \\
\hline Precisão & $100 \% \sigma 0$ & $99.66 \% \quad \sigma 0.004$ \\
\hline Revocação & $99.63 \% \quad \sigma 0.004$ & $100 \% \sigma 0$ \\
\hline F1-score & $100 \% \sigma 0$ & $100 \% \sigma 0$ \\
\hline
\end{tabular}

Alguns trabalhos na literatura alcançaram resultados expressivos na classificação de ovos de algumas espécies de parasitos. Grande parte deles utilizaram técnicas de processamento digital de imagens buscando extrair características da morfologia dos ovos para cada espécie. Os resultados obtidos ilustrados nas Tabelas I, II, III, IV e V demonstram a eficiência em utilizar CNNs para o problema em questão.

Ao se avaliar o valor de revocação médio para uma determinada espécie de ovo de parasito em cada experimento, foi obtido uma taxa de acerto de $100 \%$ para o grupo dos Ancilostomídeos, $100 \%$ para o parasito Ascaris lumbricoides, 99.50\% para Enterobius vermicularis, $98.63 \%$ para a espécie Schistosoma mansoni e uma taxa de $100 \%$ de acerto para a classificação correta dos ovos da espécie Trichuris trichiura.

A justificava de uma maior importância na avaliação do algoritmo proposto para a métrica de revocação é o fato de que, para diagnósticos médicos, o número de falsos negativos deve tender a zero, uma vez que um diagnóstico falso em relação a não doença de um determinado paciente doente, pode levar ao desenvolvimento dessa enfermidade e até mesmo ocasionar sua morte.

O estudo feito levando em consideração um problema de classificação binária, em que buscou classificar corretamente ovos de uma determinada espécie em meio de uma amostra fecal que contém milhares de impurezas e sujeiras, é de extrema relevância para essa pesquisa.

Considerando o problema multiclasse, ao se observar a Tabela VI, é possível concluir que a arquitetura de CNN proposta para esse determinado problema obteve um comportamento interessante, tendo obtido valores de acurácia, precisão, revocação e F1-score próximos de 100\%, independentemente da espécie.

Ressalta-se que para todas as simulações realizadas, tanto para os problemas de classificação binária quanto o problema de classificação multiclasse, os valores das métricas obtidas foram bem próximas, resultando num desvio padrão próximo de zero, o que valida as arquiteturas propostas.

\section{CONCLusões}

Nesse artigo foi apresentado uma nova solução para automatizar o diagnóstico de algumas espécies de parasitos intestinais humanos, são elas: Ancylostoma duodenale, Necator americanus, Ascaris lumbricoides, Enterobius vermicularis, Schistosoma mansoni e Trichuris trichiura.

Um ponto relevante que pode ser observado nesse trabalho é a utilização de técnicas de aumento de dados para que fosse possível realizá-lo. Em grande parte dos problemas que existem na área médica, assim como o desse estudo, é praticamente impossível que se tenha um número grande o suficiente de dados para que se possa trabalhar com algoritmos de aprendizado profundo. Devido a isso, utilizar essas técnicas de aumento de dados se torna imprescindível e pertinente para que se consiga resultados satisfatórios.

O método utilizado para a classificação de ovos de parasitos em imagens de microscopia óptica foram as Redes Neurais Convolucionais, em que foi definida empiricamente a melhor arquitetura para os dois problemas propostos, o de classificação binária e o de classificação multiclasse.

Foram definidas duas arquiteturas de CNN. A primeira conseguindo diferenciar os ovos de determinada espécie das impurezas contidas em uma lâmina fecal, demonstrando sua eficiência. A segunda conseguiu classificar cada ovo de espécies diferentes com uma taxa de acerto de quase $100 \%$, apesar da semelhança morfológica entre algumas espécies.

Os resultados obtidos demonstram uma classificação próxima de $100 \%$ para os problemas abordados. É importante ressaltar que o método pode ser estendido a um número maior de espécies de parasitos, seguindo procedimentos parecidos.

As Redes Neurais Convolucionais podem ser utilizadas para outros casos que contenham imagens biomédicas, como ressonância magnética de mamografia, imagens de microscópio ou de satélites. Portanto, esse trabalho representa uma contribuição significativa para automatizar o diagnóstico de doenças humanas intestinais e serve como embasamento para a aplicação de arquiteturas de CNNs em outros problemas.

Além disso, outros trabalhos futuros podem ser pesquisados a partir do estudo aqui desenvolvido, tais como: realizar a coleta de mais imagens por meio de diferentes microscópios com o objetivo de verificar se não há enviesamento pela fonte da captura das imagens, testar com pacientes de regiões que haja alta incidência de alguma espécie em específico entre outros trabalhos.

O objetivo final desse trabalho é proporcionar um algoritmo baseado em CNN que sirva como base para o desenvolvimento de um sistema computacional totalmente automatizado para a análise de amostras fecais inclusive, podendo ser aplicado 
Tabela VI

RESULTADOS DOS EXPERIMENTOS REALIZADOS COM OS OVOS DE TODAS AS ESPÉCIES ESTUDADAS NESSA PESQUISA.

\begin{tabular}{|c|c|c|c|c|c|}
\cline { 2 - 6 } \multicolumn{1}{c|}{} & Ancilosmídeos & $\begin{array}{c}\text { Ascaris } \\
\text { lumbricoides }\end{array}$ & $\begin{array}{c}\text { Enterobius } \\
\text { vermicularis }\end{array}$ & $\begin{array}{c}\text { Schistosoma } \\
\text { mansoni }\end{array}$ & $\begin{array}{c}\text { Trichuris } \\
\text { trichiura }\end{array}$ \\
\hline Acurácia & \multicolumn{5}{|c|}{$99.46 \% \sigma 0.021$} \\
\hline Precisão & $99.63 \% \sigma 0.010$ & $100 \% \sigma 0$ & $99.66 \% \sigma 0.011$ & $99.90 \% \sigma 0.005$ & $98.80 \% \sigma 0.038$ \\
\hline Revocaçãa & $100 \% \sigma 0$ & $100 \% \sigma 0$ & $98.16 \% \sigma 0.059$ & $99.66 \% \sigma 0.010$ & $99.56 \% \sigma 0.013$ \\
\hline F1-score & $99.86 \% \sigma 0.004$ & $100 \% \sigma 0$ & $98.80 \% \sigma 0.034$ & $99.76 \% \sigma 0.006$ & $99.16 \% \sigma 0.020$ \\
\hline
\end{tabular}

no Sistema Único de Saúde (SUS). Dois diferentes tipos de sistemas são possíveis. Um sistema on-line em que o microscópio é conectado a um servidor remoto e com isso, o servidor possa ter acesso a imagem e conseguir fazer a detecção dos ovos. E uma opção alternativa seria um sistema embarcado totalmente acoplado ao microscópio óptico que poderia realizar a detecção dos ovos de forma automática. O algoritmo apresentado nesse trabalho será parte essencial de um futuro sistema que permitirá automatizar a detecção de ovos de parasitos para o diagnóstico em exames fecais.

\section{REFERÊNCIAS}

[1] C. G. Schluth, C. J. Standley, S. Bansal, and C. J. Carlson, "Mapping the human helminthiases: advances and gaps in neglected disease surveillance," MedRxiv, 2020.

[2] T. C. Gomes, M. F. Almeida, L. A. Miura, J. Granja, D. V. Santos, R. M. Oliveira, A. Lopes, B. P. Sequeira, A. A. Rolemberg, A. L. Moraes et al., "Helmintoses intestinais em população de rua da cidade do rio de janeiro," Rev Soc Bras Med Trop, vol. 35, no. 5, pp. 531-2, 2002.

[3] C. J. Kucik, G. L. Martin, and B. V. Sortor, "Common intestinal parasites." American family physician, vol. 69, no. 5, 2004.

[4] G. A. De Carli, "Parasitologia clínica: seleção de métodos e técnicas de laboratório para diagnóstico das parasitoses humanas," in Parasitologia clínica: seleção de métodos e técnicas de laboratório para diagnóstico das parasitoses humanas. Coleciona SUS, 2001, pp. 810-810.

[5] D. P. Neves, "Parasitologia dinâmica," in Parasitologia dinâmica. Coleciona SUS, 2003, pp. 474-474.

[6] B. A. S. Oliveira, A. P. De Faria Neto, R. M. A. Fernandino, R. F. Carvalho, A. L. Fernandes, and F. G. Guimarães, "Automated monitoring of construction sites of electric power substations using deep learning," IEEE Access, vol. 9, pp. 19 195-19207, 2021

[7] Y. S. Yang, D. K. Park, H. C. Kim, M.-H. Choi, and J.-Y. Chai, "Automatic identification of human helminth eggs on microscopic fecal specimens using digital image processing and an artificial neural network," IEEE Transactions on Biomedical Engineering, vol. 48, no. 6, pp. 718-730, 2001.

[8] E. Dogantekin, M. Yilmaz, A. Dogantekin, E. Avci, and A. Sengur, "A robust technique based on invariant moments-anfis for recognition of human parasite eggs in microscopic images," Expert Systems with Applications, vol. 35, no. 3, pp. 728-738, 2008.

[9] D. Avci and A. Varol, "An expert diagnosis system for classification of human parasite eggs based on multi-class svm," Expert Systems with Applications, vol. 36, no. 1, pp. 43-48, 2009.

[10] C. T. Suzuki, J. F. Gomes, A. X. Falcao, J. P. Papa, and S. HoshinoShimizu, "Automatic segmentation and classification of human intestinal parasites from microscopy images," IEEE Transactions on biomedical Engineering, vol. 60, no. 3, pp. 803-812, 2012.

[11] C. T. Suzuki, J. F. Gomes, A. X. Falcao, S. H. Shimizu, and J. P. Papa, "Automated diagnosis of human intestinal parasites using optical microscopy images," in 2013 IEEE 10th International Symposium on Biomedical Imaging. IEEE, 2013, pp. 460-463.

[12] O. T. Nkamgang, D. Tchiotsop, B. S. Tchinda, and H. B. Fotsin, "A neuro-fuzzy system for automated detection and classification of human intestinal parasites," Informatics in Medicine Unlocked, vol. 13, pp. 8191, 2018.

[13] O. T. Nkamgang, D. Tchiotsop, H. B. Fotsin, P. K. Talla, V. L. Dorr, and D. Wolf, "Automating the clinical stools exam using image processing integrated in an expert system," Informatics in Medicine Unlocked, vol. 15 , p. 100165, 2019.
[14] B. S. Tchinda, M. Noubom, D. Tchiotsop, V. Louis-Dorr, and D. Wolf, "Towards an automated medical diagnosis system for intestinal parasitosis," Informatics in Medicine Unlocked, p. 100238, 2019.

[15] D. H. Hubel and T. N. Wiesel, "Receptive fields, binocular interaction and functional architecture in the cat's visual cortex," The Journal of physiology, vol. 160, no. 1, pp. 106-154, 1962.

[16] Y. LeCun, Y. Bengio et al., "Convolutional networks for images, speech, and time series," The handbook of brain theory and neural networks, vol. 3361, no. 10, p. $1995,1995$.

[17] Y. Bengio, I. Goodfellow, and A. Courville, Deep learning. MIT press Massachusetts, USA:, 2017, vol. 1.

[18] C. Szegedy, W. Liu, Y. Jia, P. Sermanet, S. Reed, D. Anguelov, D. Erhan, V. Vanhoucke, and A. Rabinovich, "Going deeper with convolutions," in Proceedings of the IEEE conference on computer vision and pattern recognition, 2015, pp. 1-9.

[19] Y. LeCun, K. Kavukcuoglu, and C. Farabet, "Convolutional networks and applications in vision," in Proceedings of 2010 IEEE International Symposium on Circuits and Systems. IEEE, 2010, pp. 253-256.

[20] C. M. Bishop, Pattern recognition and machine learning. springer, 2006

[21] A. G. Howard, M. Zhu, B. Chen, D. Kalenichenko, W. Wang, T. Weyand, M. Andreetto, and H. Adam, "Mobilenets: Efficient convolutional neural networks for mobile vision applications," arXiv preprint arXiv:1704.04861, 2017.

[22] F. Chollet, "Xception: Deep learning with depthwise separable convolutions," in Proceedings of the IEEE conference on computer vision and pattern recognition, 2017, pp. 1251-1258.

[23] G. Huang, Z. Liu, L. Van Der Maaten, and K. Q. Weinberger, "Densely connected convolutional networks," in Proceedings of the IEEE conference on computer vision and pattern recognition, 2017, pp. 4700-4708.

[24] Z. Ting-Jun, Z. Chang-Hai, X. Long-Qi, Z. Bin, X. Yan-Hong, and C. Ying-Dan, "Interpretation of detection of intestinal helminthes-the kato-katz method (ws/t 570-2017)," Zhongguo xue xi chong bing fang zhi za zhi= Chinese journal of schistosomiasis control, vol. 30, no. 5, pp. 575-577, 2018.

[25] F. Chollet et al., "Keras," 2015. 